\title{
Germanica
}

\section{Être seul et être au monde : à propos du Testament et des Lettres françaises à Merline de Rainer Maria Rilke}

Alleinsein und In-der-Welt-Sein : Zu Rilkes Testament und Lettres françaises

à Merline

\section{Arlette Camion}

\section{OpenEdition}

\section{Journals}

Édition électronique

URL : http://journals.openedition.org/germanica/1326

DOI : 10.4000/germanica.1326

ISSN : 2107-0784

Éditeur

Université de Lille

\section{Édition imprimée}

Date de publication : 30 juin 1998

Pagination : 109-122

ISBN : $9770984263203-22$

ISSN : 0984-2632

\section{Référence électronique}

Arlette Camion, « Être seul et être au monde : à propos du Testament et des Lettres françaises à Merline de Rainer Maria Rilke », Germanica [En ligne], 22 | 1998, mis en ligne le 31 janvier 2012, consulté le 06 octobre 2020. URL : http://journals.openedition.org/germanica/1326 ; DOI : https://doi.org/10.4000/ germanica.1326

Ce document a été généré automatiquement le 6 octobre 2020.

(c) Tous droits réservés 


\title{
Être seul et être au monde : à propos du Testament et des Lettres françaises à Merline de Rainer Maria Rilke
}

\author{
Alleinsein und In-der-Welt-Sein: Zu Rilkes Testament und Lettres françaises \\ à Merline
}

Arlette Camion

1 Dans un siècle où la découverte et l'aventure de soi semblent devoir nécessairement faire le détour par l'Autre, Rilke va réclamer la solitude comme un bien, comme la condition même de ce qu'il nomme depuis les années passées sous l'influence de Rodin son «travail ». Solitude non pas subie comme la malédiction frappant le poète, qui, tel le Tonio Kröger de Thomas Mann envierait la bien heureuse communion des «gens ordinaires ", mais au contraire solitude revendiquée, recherchée, cultivée. Solitude qui n'est pas un acte « contre », mais un acte " pour », non pas repli mais ouverture.

Durant les années de guerre c'est à la solitude mauvaise et au silence forcé que Rilke avait été confronté. Le flot interrompu des Élégies était resté comme l'exigence toujours présente et insatisfaite, toile de fond à la succession des jours. Après l'explosion de 1912 à Duino, l'inspiration avait repris, en 1915, pour tarir rapidement. Rilke savait que c'était là son œuvre et qu'il ne pouvait s'y dérober. Dans sa correspondance revient sans cesse la plainte: une cassure s'est produite dans son existence, et c'est à lui de renouer le fil de sa vie. Ce qui ne veut dire qu'une chose : non pas renouer avec la vie comme diversité d'événements et de rencontres, mais renouer avec sa propre solitude et son propre silence. Rilke va penser un moment ne pas pouvoir y parvenir. Ce moment coöncide avec une rencontre justement, dont il saura apprécier l'importance et mesurer les conséquences qu'elle pouvait avoir : en août 1919 une première fois, puis en août de l'année suivante, Rilke revoit à Genève une connaissance parisienne, Madame Klossowska, sous son nom de peintre, Baladine. Tous deux vont être surpris par la soudaineté de leur passion. Rilke très vite va réclamer l'impossible éloignement. La correspondance à celle qu'il appelle Merline témoigne de la crise que cet amour provoque. Ecrites en grande partie en français, qui n'était la langue maternelle ni de 
l'un ni de l'autre, les «Lettres à Merline » ont été publiées après la mort du poète avec l'accord de Madame Klossowska. Une œuvre étrange de la même période, Le Testament, ne sera rendue publique qu'en 1974: entre écrit strictement privé et fragment d'autobiographie, Le Testament, pose la question de la solitude à un moment où Rilke pense que son effort solitaire peut-être n'aboutira pas, qu'il aura été en quelque sorte imposé pour rien, que la tension qui devrait mener à l'avènement des Élégies n'aura été que tension pure et non attente pleine déjà de son achèvement. Les réflexions qui traversent Le Testament comme la correspondance de cette période (période qui se concluera pourtant par la miraculeuse explosion poétique de février 1922, Rilke écrit en quelques jours les Sonnets à Orphée et termine les Élégies) témoignent de l'importance de ce que le poète nomme «Einsamkeit» ou «Alleinsein ». Aux pires instants de doute la solitude est affirmée comme bien. C'est de son sein que Rilke voudrait pouvoir bâtir le rapport à l'autre.

3 Tout ce faisceau de questions occupe le poète depuis longtemps déjà. On se rappelle la récurrence du thème de l'enfant prodigue dans l'œuvre de Rilke : poèmes dédiés à cette figure, parabole reprise en guise de conclusion des Cahiers de Malte Laurids Brigge, traduction du texte de Gide, l'enfant prodigue revient comme un leitmotiv de 1902 à 1914. Le Testament se conclue lui aussi par une allusion à la figure de celui qui tombe à genoux non pour qu'on l'absolve mais pour implorer les autres « de ne pas l'aimer ». La lecture rilkéenne de la parabole biblique omet toute la problématique du pardon parce que le fils n'a rien à se faire pardonner, que le rapport à l'autre se situe non du côté de la faute, commise ou à commettre, mais tout entier dans la dimension de l'innocence et de la liberté. Ce fait est frappant: Rilke n'arrivera jamais à accepter, ni même à vraiment comprendre, la manœuvre sentimentale grâce à laquelle l'un accapare l'autre, il ressentira toujours comme une "usurpation " toute tentative de possession, et comme une intolérable emprise les tendresses envahissantes. Le poème de juin 1906, Der Auszug des verlorenen Sohnes, est tout centré sur le départ, exigence impérieuse qui coupe court au cercle vicieux de l'identité imposée, et de l'identité en général, de l'appartenance, de la gratitude mutilante. Il s'agit moins d'échapper à l'Autre qu'à la mauvaise conscience de ne pas répondre à l'attente légitime de son amour. Seul le départ est capable d'ouvrir, dans le risque et l'incertitude du sens, l'espace d'une existence qui ne soit pas consolation, mais affirmation de l'ici :

und fortzugehen : wohin? Ins Ungewisse,

weit in ein unverwandtes warmes Land,

das hinter allem Handeln wie Kulisse

gleichgültig sein wird : Garten oder Wand ;

und fortzugehen : warum? Aus Drang, aus Artung,

aus Ungeduld, aus dunkler Erwartung,

aus Unverständlichkeit und Unverstand :

Dies alles auf sich nehmen und vergebens

vielleicht Gehaltnes fallen lassen, um

allein zu sterben, wissend nicht warum (W, 1,492)

Les cahiers de Malte Laurids Brigge poursuivent la réflexion en aval: la rupture et la solitude sont données comme une condition de l'amour "intransitif ». Cet amour-là s'affirme comme une direction, qui ne nie pas son objet mais le traverse pour s'élancer plus loin. Or, le texte des Cahiers est sur ce point très clair, en lui réside le « travail » du poète. Travail toujours à recommencer, qui ne peut se prévaloir d'un « résultat ». Plus tard Rilke le nommera parole orphique. Celle-ci se soutient certes du désir, mais d'un 
désir qui ne s'épuise pas dans le désir de l'autre ni ne le «sublime», qui tout en le vivant le dépasse :

Gesang, wie du ihn lehrst, ist nicht Begehr,

nicht Werbung um ein endlich noch Erreichtes [...]

In Wahrheit singen ist ein anderer Hauch.

Ein Hauch um nichts. Ein Wehn im Gott. Ein Wind. (W, I, 732)

5 Il faut comprendre Rilke comme il voulait être compris, et à l'époque qui nous occupe être compris de Madame Klossowska : il ne s'agit pas d'un refus d'être aimé ou d'aimer, mais d'une exigence, exorbitante certes pour l'amante, celle d'aimer au-delà de la possession, de porter plus loin l'élan que l'amour donne. Car l'amour est généreux, il est profusion. Dans une lettre de décembre 1920 Rilke a pour Merline ces mots de profonde reconnaissance :

... oh! si jamais avec cette voix qui est déjà en toi, avant qu'elle ne soit sortie de ma bouche, je pouvais te raconter l'Histoire de notre amour, tu serais transportée d'un étonnement de félicité...; car c'est encore tellement plus beau que nous ne le devinons; notre souvenir, si agrandi par la moisson multiple de cette année de bénédiction, ne suffit pourtant pas à contenir toute la récolte : les trois-quarts, soisen sûre, sont restés dehors, en plein vent... (LM, 63)

Merline ne s'y trompe pas : elle voit parfaitement la situation de concurrence déloyale où elle se trouve mise à son insu. Car Rilke lui oppose son «travail » auquel il doit son « amour » davantage qu'à l'amante. Dans Das Testament, la position est très claire : « Das Prinzip meiner Arbeit ist eine leidenschaftliche Unterwerfung unter den Gegenstand, der mich beschäftigt, dem, mit anderen Worten, meine Liebe gehört» $(T, 39)$. Merline, elle, écrit : «Vous n'avez pas besoin de moi pour votre vie, c'est cela qui est la vérité tandis que pour moi, vous êtes toute ma vie ${ }^{1} »$. Elle est sans doute à la fois dans le vrai et dans le faux : dans le vrai car Rilke n'est prêt à accepter aucune des « usurpations de l'amour » et dans le faux car Rilke ne veut pas se priver de cet amour-là. Ne serait-ce parce que s'en préserver témoignerait de plus de lâcheté que de sagesse :

Wenn ich der Liebenden nicht widerstand, so wars, weil von allen Bemächtigungen eines Menschen über einen anderen, die ihre allein, ihre unaufhaltsame, mir im Recht zu sein schien. Ausgesetzt wie ich bin, wollte ich auch sie nicht vermeiden; aber ich sehnte mich, sie zu durchdringen! Daß sie mir Fenster sei in den erweiterten Weltraum des Daseins... (nicht Spiegel) (T, 24)

Double trahison pour la femme aimée, qui ne peut que souffrir d'être certes aimée, mais seulement pour l'élargissement de l'être que son amour apporte. Et pour l'amant certitude que la vraie trahison serait dans le repli sur l'aventure amoureuse, sa stérilité, sa confusion. Les mots du Testament sont ici très durs :

So erscheint das Liebesverhältnis als eine gleichsam verkümmerte, unfähige Nebenform der schöpferischen Begabung, als ihre Herabsetzung, - und bleibt ungekannt, unbeherrscht und, an der höheren Ordnung jenes Gelingens gemessen, unerlaubt. $(\mathrm{T}, 39)$

8 Il y a plusieurs façons dejuger cette attitude: on peut y voir un refus du corps de l'Autre, une peur de son contact, l'éloignement imposé cachant alors une faiblesse. Rilke pourtant n'a pas eu l'appréhension de l'amour physique, il y a vu au contraire le centre rayonnant d'une joie qui unit l'être au monde. Dans un texte de1922, Der Brief des jungen Arbeiters, il fait exprimer à un jeune ouvrier sa propre exécration du mépris chrétien pour la " chair » : la chair est profondément bonne, comme le terrestre est audelà de la souffrance même, profondément juste. L'Église, en attachant le péché à la 
sexualité, a introduit le trouble dans le rapport au corps, qui devrait être lumière et force :

Und hier in jener Liebe, die sie mit einem unerträglichen Ineinander von Verachtung, Begierlichkeit und Neugier die « sinnliche » nennen, hier sind wohl die schlimmsten Wirkungen jener Herabsetzung zu suchen, die das Christentum dem Irdischen meinte bereiten zu müssen. Hier ist alles Entstellung und Verdrängung, obwohl wir doch aus diesem tiefsten Ereignis hervorgehen und selber in ihm die Mitte unserer Entzückungen besitzen. Es ist mir, wenn ich es sagen darf, immer unbegreiflicher, wie eine Lehre, die uns dort ins Unrecht setzt, wo die ganze Kreatur ihr seligstes Recht genießt, in solcher Beständigkeit sich, wenn auch nirgends bewähren, so doch weithin behaupten darf. (W, VI, 1123)

Ni rejet, ni effroi du corps de l'autre ne sont ici la cause secrète qui commande le recul. Serait-ce alors la pure incapacité d'accepter l'engagement quotidien que nécessite l'amour, dans sa banale, la décevante humanité ? C'est la conclusion que tire Michel Vanoosthuyse de sa lecture de la parabole du fils prodigue à la fin des Cahiers de Malte Laurids Brigge: "on aura peine à me persuader ", dit-il, "que l'histoire de l'enfant prodigue ainsi revue ne soit pas en fin de compte l'histoire du marginal flamboyant quoi qu'encore douloureux, qui refuse les contraintes de la vie pratique et de la vie sociale...; de celui qui est délié; qui participe à la vie cosmique, mais qui ne se reconnaît aucune obligation extérieure envers ce qui pourrait l'attacher à un autre en particulier; qui évite de se nouer au regard de l'autre et de se laisser nouer par lui, parce que ce regard enferme et aliène $»^{2}$. Le reproche touche juste, certainement. Mais il touche l'homme Rilke, dans son évidente incapacité à se lier durablement si ce n'est dans la distance que procure le commerce épistolaire. Il ne touche pas le poète et sa conception de «l'amour»: celle-ci est davantage, infiniment, que le simple aveu de l'impuissance à accepter les inévitables contraintes de ce qu'on nomme les relations humaines.

On pourra objecter à nouveau que dans le cas de Rilke le poète et l'homme sont on ne peut plus proches. Que tout l'effort de l'homme est de mener une existence dans la poésie, et que le poète chante du sein même de son existence d'homme. C'est juste, là encore, et à nuancer à nouveau. Dans une des Lettres à Merline, écrite du château de Berg am Irchel près de Zurich, prêté par des amis pour une retraite, Rilke précise avec une pointe d'irritation que son travail n'est pas " production » mais « disponibilité ». C'està-dire qu'il lui est intimement lié, lié au plus profond de son existence. Comme souvent dans les lettres les plus graves, Rilke passe à la langue maternelle qu'il partage en ces instants-là avec l'amante :

Laß mich nun, Liebste, laß mich die nächsten Monate, solang diese Zuflucht vergönnt ist, mein Leben ordnen und klären. (Ich kann nicht bestehen bleiben in dieser jahrelangen Trübe !) : darum handelt es sich ja für mich nicht um die Elegien oder sonst ein Produktiv-Werden - ich bin ja kein « Autor ", der « Büchermacht ». Selbst die Elegien (oder was mir eines Tages gewährt würde) waren ja nur eine Folge einer inneren Verfassung, eines inneren Fortschritts, eines ReinerUmfassendergewordenseins meiner ganzen unterbrochenen und verschütterten Natur... Versteh mich : so sehr ich meiner Arbeit gehör und diene - ich kann sie nicht hervorrufen - darum ergriff mich das Wort bei Bonsels so, daß keiner je mehr erreicht habe, als Äußerstes, als die «Bereitschaft "- um Bereitschaft ringe ich jetzt, da darf mich keiner anrühren oder erschüttern, denn sie ist wie die Entstehung eines Kristalls abhängig von den entferntesten Einflüßen, die uns nur erreichen, wenn wir in der Konstellation stehen und unverschoben von Zufall, Willkür, Begier oder Widerstand ! Oh, meine Liebe, glaub mir daß ich die «Welt » weiß, daß ich nichts will als « im Gesetz bleiben » $(\mathrm{LM}, 95)$ 
11 Et Rilke ajoute, comme pour convaincre encore la femme aimée que son amour n'est pas le rival de l'autre, de l'amour plus grand : « wie sollte Dir das irgendwann Schaden thun können? Wollen wir denn von unserer Liebe andere Erweisungen und Wohlthaten, als jene die auf dem gleichen Gesetze beruhen : wehe der Liebkosung, die den Sternen entgegen ist ! ». L'amant se confond donc avec le poète mais sans que le second veuille «tirer profit » du premier : Rilke n'est pas «inspiré » par son amour pour Merline, il n'entend pas exploiter poétiquement l'amour. Au contraire, il veut subordonner l'amour pour l'amante à la "loi du monde", à l'amour impersonnel, anonyme. En lui l'affrontement du Moi et du Toi, ou leur conjonction, leur étreinte même, ne sont plus ce qui importe. Le malentendu persistera tant que la femme aimée comprendra comme de l'égoïsme ce qui est au contraire un effort chez le poète pour exprimer autre chose que les simples mouvements de son âme, et comme une esquive ce qui est un effort chez l'homme pour ne pas trahir le poète. Il semble que Merline, avec difficultés, se soit rangée aux raisons de Rilke : elle était elle-même artiste, femme intelligente, mère attentive des enfants qui deviendront Pierre Klossowski et Balthus. Et pourtant Le Testament témoigne que Rilke a souffert de cette relation dont il savait qu'elle aurait pu être "sublime »: «Daß alles herrlich ist zwischen uns - weißt Du's nicht » s'étonne-t-il dans les lettres (LM, 97), tandis que dans Le Testament, il avoue de celles reçues de Merline: "Briefe : wie war ich hin und hergerissen diesen Winter; jeder Brief ein Stoß, ein Angriff, der alles umstürzen konnte, oder eine innige Eindringung, die das Blut verwandelte -» $(T, 27)$.

12 Comment définir à présent l'autre amour que Rilke, nomme aussi «travail », ou "disponibilité », comment saisir vraiment ce qui n'annule pas, mais excède toute relation humaine ? C'est la poésie conçue comme un au-delà du lyrisme sentimental, et le poète est authentique lorsqu'il s'est oublié, lorsque son chant n'est plus l'expression de lui-même, mais recueil du chant diffracté dans choses, êtres, lieux, événements. Le 27 octobre 1919 Rilke introduit à Zurich la lecture de ses poèmes par un bref exposé dans lequel il cherche à définir ce dépassement du « lyrisches Ich » :

(Das Werk aus dem er nun lesen werde, entspringe der Überzeugung, daß seine Sendung darin liege), daß es eine eigene berechtigte Aufgabe sei, die Weite, Vielfältigkeit, ja Vollzähligkeit der Welt in reinen Beweisen vorzuführen. Denn : ja ! zu einem derartigen Zeugnis hoffte ich mir das Gedicht zu erziehen, das mir fähig werden sollte alle Erscheinung, nicht nur das Gefühlmäßige allein, lyrisch zu begreifen: Das Tier, die Pflanze, jeden Vorgang; - ein Ding in seinem eigentümlichen Gefühls-Raum darzustellen ${ }^{3}$.

13 Depuis les Neue Gedichte la conception n'a pas changé. Et le plan d'ensemble des Élégies, fixé dès le début, en sera l'aboutissement: de la plainte à la célébration, l'homme dépasse la limitation de son séjour terrestre pour en affirmer la plénitude. Et c'est parce qu'il s'oublie dans "l'être", si le mot ne fait pas peur, que disparaît la douloureuse confrontation objet/sujet, moi/monde, moi/toi, comme toutes les autres partitions mutilantes. Rilke a refusé de se soumettre à une cure psychanalytique dont à un moment il ressentait le besoin parce que justement cette thérapie renforce les partitions binaires, les oppositions, les rapports de face-à-face. La psychanalyse ressasse la question de l'identité, profondément étrangère à Rilke. L'amour au quotidien aussi, peut-être, c'est en tous cas l'appréhension que le poète ressent lorsqu'il dit vouloir de l'amante qu'elle soit non miroir mais fenêtre. L'amour plus grand que celui qui s'épuise et s'abîme dans l'autre est la pleine acceptation de ce qui est. L'affirmation du terrestre, de la pure immanence avec la douleur, le mal et la mort, 
dans la conscience même de la mort : c'est parce qu'Orphée est revenu des Enfers que son chant est valide, que son chant est juste. Il n'est en rien consolation, car, Rilke ledit à Merline, «que nous servirait une vie consolée?» (LM, 46). Il n'occulte rien de l'insoutenable, sans se faire pourtant dans la dimension de la perte. Rühmen, preisen, loben sont les verbes pivots des Sonnets à Orphée comme des Élégies. Plus de place ici pour aucune des stratégies amoureuses de possession, de consolation, de complaisance spéculaire. On peut se moquer de l'amour cosmique rilkéen, bien sûr, et lui préférer le dur labeur de l'autre, le dévouement ingrat, l'attention minuscule et grandiose à la fois que l'on porte à l'autre visage. Là aussi est l'oubli de soi, ce que Rilke n'a jamais vraiment saisi. Cependant on ne peut taxer de ruse, de faiblesse ou de lâcheté la « célébration » de l'ici qui dépasse la circularité de la relation amoureuse.

Lorsque Merline se désespère de son absence, il lui répond : "Suis-je donc condamné à vous faire tant, tant souffrir? Je vous en supplie, une petite trêve de douleur; regardez : c'est encore la vie, la même qui nous a portés sur les sommets de nos cœurs, vous ne pouvez pas l'accuser de cruauté sans lui reprocher en même temps d'avoir été si généreuse » (LM, 23), ou encore « Dites, vous sou-tiendrais-je si mal que, malgré toute mon assistance amoureuse, vous vous laissiez choir dans une si terrible mécréance de la vie? » (LM, 27) Merline finit par comprendre, mais il n'est pas sûr que Rilke lui ait donné à lire Le Testament. Le manuscrit sera détenu par l'ami et éditeur Anton Kippenberg et par la grande confidente de toutes ces années, Nanny Wunderly-Volkart, plus tard par les héritiers du poète. En vertu d'une sorte d'accord tacite, l'œuvre restera inédite jusqu'en 1974. Car son enjeu est à mi-chemin entre l'ordre strictement privé et la problématique plus générale qui est celle de l'amour de la femme contre l'amour du monde ${ }^{4}$. L'homme Rilke veut défendre le poète au moment où le poète n'est plus sûr de rien : la retraite de Berg am Irchel s'avère avoir été un échec. L'épreuve n'a pas été la solitude, mais sa stérilité. La solitude n'a pas été " die Speerspielerin », celle qui projette au loin la parole du poète comme une lance ( $T, 22)$. À l'époque des Neue Gedichte et des Cahiers de Malte Laurids Brigge, Rilke croyait à l'éthique du travail, à l'application du poète sur son matériau comme à celle du sculpteur sur sa pierre. Il avait fait sienne alors la devise de Rodin «travailler, toujours travailler ", il voyait dans le labeur obscur et quotidien le seul salut, la seule grandeur. À partir de 1910-1912 ses conceptions se modifient du tout au tout: ce n'est pas le travail (bien que le mot reste synonyme chez lui de son activité poétique) qui prime, il n'y a nul " progrès ", mais une attente. Dans la lettre du 20 février 1921, Rilke tente de l'expliquer à Merline :

Dans ma lettre d'hier vous avez pu vous convaincre que je suis loin, loin du travail. Je ferai tout mon possible pour m'approcher, mais si j'arrive lentement par une discipline rigoureuse de tous les jours, même touchant au travail, je serai encore et pour longtemps - loin de cette tâche suprême. Je ne peux employer aucune ruse, ni même aucun effort direct pour pénétrer dans cette sphère ineffable qui ne m'était jamais accessible qu'après un temps de soumission absolue et d'une obéissance quotidienne, employée d'abord à bien exécuter les ordres d'une valeur secondaire. Ce ne serait plus ma solitude si je voulais vous en découvrir toutes les lois - mais il m'est permis de vous confier que j'aurai un long chemin à faire pour être là où on commence. (LM, 82)

L'attente de ce commencement, du mouvement par où la parole poétique s'inaugure est à la fois tension, joie d'être au monde, recueil déjà de ce qui va être dit. «Wovon sollte je Kunst ausgehen, wenn nicht von dieser Freude und Spannung unendlichen Anbeginns?» (LM, 88). Or cette tension ne se fait que dans la solitude qui ouvre au monde, la solitude est déjà être au monde, elle est déjà attitude poétique. La parole 
poétique vient de surcroît, alors que l'on ne l'attendait plus, comme un cadeau inespéré. En elle ne se dit pas la présence du poète aux choses, celle-ci est déjà dans l'attente solitaire, mais la présence des choses. Pour être plus précis il faudrait parler ici plutôt que d'attente, d'écoute. Orphée avant de chanter écoute, entend du sein du silence. Et le poète recueille, s'il sait entendre par-delà le vacarme des jours, les bribes du chant orphique: trace et non perte, né de la disparition, mais affirmant la persistance, le chant orphique est non ce qui reste après le retrait du Dieu, du sens, mais ce qui s'entend du sein de cette disparition même. Le célèbre sonnet 26 de la première partie des Sonnets à Orphée va servir de guide à un autre grand solitaire, le peintre Cy Twombly :

O du verlorner Gott ! Du unendliche Spur,

nur weil dich reißend zuletzt die Feindschaft verteilte

sind wir die Hörenden jetzt und ein Mund der Natur. (I, 748)

L'attente solitaire est l'indispensable silence qui permet d'entendre. Elle crée cette dimension paradoxale, qui est à la fois proximité et distance. Rilke a toujours été très sensible à la brutalité avec laquelle par inintelligence l'adulte détruit la juste distance qui le sépare et le relie à la fois au monde. L'enfant, lui, a la préscience de ce juste rapport, il vit « en figures » ${ }^{5}$. L'autre est inaccessible et proche à la fois pour l'enfant, et le poète a pour tâche de saisir à nouveau le mystère de cette familière étrangeté. « Alles ist weit -, und nirgends schließt sich der Kreis » $(1,764)$ : l'effort est précisément de maintenir cette ouverture, de vivre en elle, d'être à l'écoute de sa vibration. L'attente solitaire est déjà l'être dans l'Ouvert.

Dans Le Testament Rilke fait allusion à sa traduction de Mallarmé $(\mathrm{T}, 8)$; il avait en effet dans ces années qui précèdent immédiatement l'achèvement des Élégies et Les sonnets à Orphée traduit L'Éventail de mademoiselle Mallarmé et Tombeau. On ne s'étonnera pas de son admiration pour le poète français qui déclarait : « La poésie est l'expression, par le langage humain, ramené à son rythme essentiel, du sens mystérieux des aspects de l'existence: elle doute ainsi l'authenticité de notre séjour et constitue la seule tâche spirituelle $»^{6}$. Mais chez Mallarmé la poésie est rite, rituel, elle est principe ordonnateur de notre existence dans le sens où elle la soumet aux "purs motifs rythmiques de l'Être $»^{7}$. Il n'est pas sûr que la conception rilkéenne de la parole poétique soit comparable. Avant le chant prime l'étal de silence et davantage que la scansion de l'Être mallarméenne, c'est la montée conjointe du chant et de l'arbre, du désir et de l'oiseau qui fait être à un nouvel espace. Ainsi le premier des Sonnets à Orphée s'ouvre-til sur ce mouvement :

Da stieg ein Baum. O reine Übersteigung!

o Orpheus singt ! o hoher Baum im Ohr!

Und alles schwieg. Doch selbst in der Verschweigung

ging neuer Anfang, Wink und Wandlung vor. (I, 731)

Dans ce travail d'attente, qui est déjà être-au-monde tel que Rilke l'entend, le rapport à l'autre est d'une importance capitale. La solitude rilkéenne est étrangement peuplée : au moment où tout l'effort est porté vers une raréfaction du commerce avec les hommes, la correspondance témoigne au contraire d'un intense besoin d'être en relation avec les autres, l'autre. La correspondance rilkéenne fait partie de l'œuvre: non seulement parce qu'elle y prépare, mais parce que le soin apporté à la rédaction des innombrables missives à d'innombrables correspondants en fait souvent de vrais textes. Pourquoi tant écrire, se demande parfois Rilke lui-même? Pour de multiples raisons : d'abord parce qu'écrire rompt le "grand mutisme », « die große Stummheit » 
des années de guerre. Avec frénésie Rilke remet à jour sa correspondance après 1919 : de Soglio en août et septembre 1919 il envoie des centaines de feuillets dans l'Europe entière. En décembre 1920, de Berg am Irchel, il écrit 115 lettres en un mois (LM, 56). Le déraciné trouve aussi par les liens épistolaires une patrie qu'il peuple des hommes et des femmes qu'il estime (et qui lui viennent en aide) : à Nanny Wunderly-Volkart il avoue : «Gott weiß, wozu ich so viele Beziehungen unterhalte, manchmal denk ich, es ist ein Ersatz für die Heimat, als ob doch eine Art von fein verteiltem Irgendwo-Sein gegeben sei, mit diesem ausgedehnten Netz von Einflüßen », tout en ajoutant aussitôt : «aber die Briefe sind auch da für die Sache, für das, was meine Arbeit ist, - schließlich schwingt doch in allen Briefen eine Spur ihrer Intensität und teilt sich mit $»^{8}$. À LilyZiegler il explique la fonction essentielle de la correspondance : « Die Briefe sind ein Aufräumen bis tief ins Gemüt hinein, sie geben der Feder eine Art Übergang vom Mündlichen und Mitteilenden zu der, niemandem Einzelnen mehr zugekehrten Schriftlichkeit der Arbeit ${ }^{9}$. À ceci il faut ajouter que de nombreux correspondants de Rilke avaient un véritable besoin de ses lettres, que quelques-uns lui écrivaient du sein de la détresse ou du doute et que le poète était incapable de laisser une lettre à lui envoyée sans réponse. Ainsi l'attente solitaire résonne-t-elle de voix lointaines et confidentes, et ces destins offerts dans l'écriture nourrissent la parole poétique. L'exemple le plus éclatant est la correspondance avec Madame Knoop qui précède immédiatement la rédaction de Sonnets à Orphée : la mort de la jeune Wera Knoop, une amie de Ruth Rilke, a profondément touché le poète. Il s'enquiert auprès de sa mère des circonstances de la disparition, et dans une succession de lettres il se confie pour sa part avec une franchise étonnante à l'égard de quelqu'un qu'il ne connaît qu'à peine. Les lettres sont donc plus que de simples moyens de communiquer des nouvelles ou d'appeler à l'aide, elles sont la création d'une chambre d'écho, d'un espace de résonnance dans lequel, ensuite, la parole poétique vient s'inscrire. Le commerce épistolaire crée sans doute cette "juste distance» que la relation amicale ou amoureuse ne peut toujours tenir.

Solitude habitée des autres, l'être-seul de Rilke n'est pas douloureux, ne connaît ni la rancœur, ni l'amertume. La solitude n'est pas un défi, pas une épreuve, mais un état qui participe du " travail sans but ", « die ziellose Arbeit » par quoi Rilke définit à la fin des Cahiers l'activité du poète ${ }^{10}$. Et lorsque le «travail» se dérobe, qu'il est encore infiniment loin, comme dans ces jours de doute des années 1920-21, Rilke conserve la certitude que la solitude est profondément nécessaire, qu'elle est la " première, la plus silencieuse et la plus libre de ses décisions » :

Manchmal in der unaufhörlich prüfenden Noth dieser Tageüberrascht mich etwas wie der vorauseilende Schein einer neuengeistigen Freude: als ob doch Alles einfacher geworden sei und einunsägliches Schicksal in Annäherungswerthen sich faßlichermache. Denn ist es schließlich nicht dies (wenn man es aussprechen soll) : daß Helle und Dunkelheit in meinem Inneren nicht durch eines Menschen überwiegenden Einfluß bestimmt werden dürfen, sondern allein durch ein Namenloses. Dies ist, sozusagen, das Mindestmaß meiner Frömmigkeit: es aufgebend, müßte ich hinter den ersten Kreuzweg meines Lebens zurück -, hinter seinefrüheste stillste freieste Entscheidung. Hinter mich selbst. (T, 50). 


\section{ANNEXES}

W : Werke in sechs Bänden, insel taschenbuch, Francfort, 1987.

LM : Lettres françaises à Merline, Seuil, Paris, 1950.

T : Das Testament, Suhrkamp, Francfort, 1976.

\section{NOTES}

1. Cité par Donald Prater, Ein klingendes Glas - Das Leben Rainer Maria Rilkes, Rowohlt, Hambourg, 1989, p. 549.

2. Michel Vanoosthuyse, L'abject et le sublime in Rainer Maria Rilke et les Cahiers de Malte Laurids Brigge - Ecriture romanesque et modernité, Masson, Paris, 1996, p. 145.

3. Cité par Donald Prater, op. cit., p. 511.

4. Voir à ce propos le Nachwort d'Ernst Zinn à l'édition de Das Testament,Suhrkamp, Francfort, 1976, p. 70.

5. On peut se reporter à mon article Une lecture du poème "Enfance» de Rainer Maria Rilke, Germanica 15, Lille, 1994, p. 35.

6. Mallarmé, Correspondance II, 1871-1885, Gallimard, Paris, 1965, p. 266.

7. Mallarmé, Crayonné au théâtre, Euvres Complètes, La Pléiade, Paris, p. 345.

8. Cité par Donald Prater, op. cit., p. 520.

9. Ibidem, p. 538.

10. Werke I, p. 943.

\section{RÉSUMÉS}

Le Testament, œuvre qui restera inédite jusqu'en 1974 et les Lettres françaises à Merline témoignent de la crise grave qui affecte Rilke à un moment où, en tant que poète, il doute de pouvoir terminer les Élégies, et où, en tant qu'homme, il est requis par son amour pour Madame Klossowska. Le court texte autobiographique comme la correspondance mettent en place toute une réflexion sur la solitude : revendiquée comme l'espace d'où doit se bâtir le rapport à l'autre, la solitude est aussi, poétiquement, l'étal de silence qui rend possible le chant. Elle est déjà écoute et pleinement présence aux choses. La solitude rilkéenne est en outre peuplée des voix multiples de la correspondance; celle-ci n'est pas seulement une préparation à l'écriture, mais aussi la patiente élaboration d'un rapport à l'autre qui ne soit pas spéculaire.

Das Testament ist ein kurzes autobiographisches Werk von Rilke, das bis 1974unveröffentlicht blieb. Die Lettres françaises à Merline ihrerseits sind eine Auswahl aus dem Briefwechsel mit der Geliebten, Madame Klossowska. Beide Bücher zeugen von der tiefen Krise, die Rilke in den Jahren vor der endgültigen Niederschrift der Duineser Elegien erlebt. Auch in den Augenblicken, da er an seiner Fähigkeit zweifelt, sein Werk vollenden zu können, wird die Einsamkeit als « die freieste aller Entscheidungen» behauptet. Von ihr aus soll die Beziehung zum anderen Menschen 
entstehen. Für den Dichter ist sie das Schweigen, aus dem « der Gesang steigt », sie ist nicht nur Warten auf das Gedicht, sondern bewusstes und volles « In-der-Welt-Sein ». Rilkes umfangreicher Briefwechsel begleitet seine selbst gewählte Einsamkeit: Das Briefe-Schreiben ist Vorbereitung auf das Dichten, und schafft auch die respektvolle Nähe und die vertraute Ferne, aus der die gelungene Beziehung zum Anderen entsteht.

\section{AUTEUR}

\section{ARLETTE CAMION}

Université d'Orléans 